\title{
Módulo de simulação realista para redes de acesso móveis LTE/LTE-A
}

\author{
Luiz R. F. Sekijima ${ }^{1}$, Tiago P. C. de Andrade ${ }^{1}$, Nelson L. S. da Fonseca ${ }^{1}$ \\ ${ }^{1}$ Instituto de Computação - Universidade Estadual de Campinas (UNICAMP) \\ Campinas - SP - Brazil \\ 1117842@g.unicamp.br, tiagoandrade@lrc.ic.unicamp.br, nfonseca@ic.unicamp.br
}

\begin{abstract}
The growth of mobile devices in recent years demands access technologies that are fast, reliable and power efficient. Simulation allows the evaluation of new algorithms for the emerging networks at low cost. This project is based on the mobile access networks Long Term Evolution (LTE) and LTEAdvanced (LTE-A) and presents a module for the LTE-Sim simulator that can be used to study several mechanisms proposed in LTE/LTE-A networks. Realistic scenarios were defined to validate the developed module and the results are in accordance with those found in the literature.
\end{abstract}

Resumo. O crescimento no uso de dispositivos móveis nos últimos anos demanda por tecnologias de acesso que sejam rápidas, confiáveis e eficiente energeticamente. A simulação permite que novos algoritmos sejam projetados $e$ avaliados para redes emergentes com baixo custo. Este projeto foca nas redes de acesso móveis Long Term Evolution (LTE) e LTE-Advanced (LTE-A) e apresenta um módulo para o simulador LTE-Sim com o objetivo de estudar diversos procedimentos propostos para redes LTE/LTE-A. Cenários foram criados para validar o módulo e os resultados encontrados são condizentes com os encontrados na literatura.

\section{Introdução}

Estima-se que no fim de 2017, já haviam 7.8 bilhões de usuários de banda larga móvel no mundo [Ericsson 2017]. Serviços de streaming de música e vídeo tornaram popular nos últimos anos, exigindo altas taxas de vazão (do inglês throughput) e baixa latência. O Third-generation Partnership Project (3GPP) é uma organização que visa o desenvolvimento de especificações para sistemas móveis, o seu escopo de trabalho inclui as redes de acesso móveis, serviços, o núcleo da rede e os terminais móveis. Em 2009, o 3GPP lançou a release $8 \mathrm{com}$ as especificações para a rede de acesso Long Term Evolution (LTE), que é a rede que faz a comunicação entre terminal móvel e a estação-base. Desde então, novas especificações foram publicadas adicionando melhorias ao padrão LTE, entre as principais estão: release 10 - que aborda o aumento de capacidade da rede e a release 13 que adiciona de novas funcionalidades e aumento de eficiência. Na release 10, foram introduzidas técnicas como agregação de portadoras através do uso simultâneo de diferentes bandas, o que permite enviar mais dados simultaneamente, o que permite o aumento da vazão, da quantidade de usuários por célula e da eficiência espectral. As redes que implementam as especificações da release 10 são conhecidas como LTE-Advanced, ou LTE-A.

Com o objetivo de otimizar o desempenho dessas redes de acesso, simuladores de redes LTE e LTE-A vem sendo implementados por grupos de pesquisas[Ikuno et al. 2010, 
Mehlführer et al. 2009, Rupp et al. 2016]. O uso de simulação é uma forma pouco custosa, simples e rápida para testar novos algoritmos e mecanismos de controle de rede. As grandes empresas na área de telecomunicações, de uma maneira geral, tem simuladores próprios que não são disponibilizados para a comunidade científica. São poucos os simuladores de código aberto que implementam toda a pilha de protocolo do LTE; a grande maioria implementa apenas a transmissão física entre o dispositivo móvel e a estaçãobase. O LTE-Sim [Piro et al. 2011] possui uma implementação mais completa do padrão LTE, incluindo mais funcionalidades como geradores de tráfego, estratégias de escalonamento e diversos componentes da rede.

A motivação deste projeto vem do fato de que redes LTE e LTE-A possuem 2.6 bilhões de usuários mundialmente e apesar da indústria na área de telecomunicações já estarem preparando a próxima geração de tecnologia de redes de acesso, o uso do LTE deve continuar a crescer e alcançar 5.5 bilhões de usuários até 2023 [Ericsson 2017]. O desenvolvimento de novos mecanismos para o LTE pode trazer benefícios tais como redução de consumo energético, impactando financeiramente tanto os fornecedores de redes LTE, quanto os usuários.

O objetivo do projeto de iniciação científica, descrito nesse artigo, é implementação e simulação de diversos mecanismos de controle de redes LTE no simulador LTE-Sim. A principal contribuição do módulo desenvolvido para o LTE-Sim, é a implementação de modelos de tráfego, um novo procedimento de acesso aleatório, funcionalidades de comunicação Machine to Machine. O módulo desenvolvido foi usado em diversos projetos e artigos do grupo de pesquisa de redes do IC/UNICAMP.

\section{Long Term Evolution/Long Term Evolution Advanced}

A rede de acesso Long Term Evolution (LTE) [Sesia et al. 2011] é uma tecnologia de redes móveis de transmissão de dados que foi projetada para suportar comutação por pacotes (Packet Switch), substituindo o padrão anterior de comutação por circuitos que necessitava de uma conexão dedicada para a comunicação. A primeira especificação do LTE [Martín-Sacristán et al. 2009] tinha como objetivo garantir: mobilidade em alta velocidade (até $350 \mathrm{~km} / \mathrm{h}$ ); maior flexibilidade e eficiência no uso do espectro; garantia de qualidade de serviço (Quality of Service - QoS), com taxa de vazão mínima e latência; redução de complexidade da rede e custo de implementação.

Os elementos que compõem o LTE são o terminal móvel, que é chamado de equipamento do usuário (User Equipment ou UE), e a estação-base, chamada evolved Node $B$ ou eNB. É o eNB que aloca recursos para que os dispositivos possam transmitir e receber dados. Foi padronizado junto com a rede de acesso o núcleo da rede, o núcleo de pacote desenvolvido (Evolved Packet Core ou EPC), responsável pelos serviços fornecidos ao usuário.

Sobre a camada física do LTE, há dois modos de operação: duplexação por divisão de frequência (Frequency Division Duplexing ou FDD) e duplexação por divisão de tempo (Time Division Duplexing ou TDD). Em modo FDD, a recepção e transmissão são realizadas em faixas de frequência distintas, enquanto em TDD, apenas uma faixa é utilizada, alternando entre transmissão e recepção. As transmissões são organizadas em frames de $10 \mathrm{~ms}$, e cada frame é dividido em dez subframes de $1 \mathrm{~ms}$, que por sua vez, são divididos em slots de $0.5 \mathrm{~ms}$. A tecnologia usada para transmissão de dados no sen- 
tido de downlink foi o múltiplo acesso por divisão de frequências ortogonais (Orthogonal Frequency-Division Multiple Access ou OFDMA) e no sentido de uplink foi o múltiplo acesso por divisão de frequências de portadora única (Single-Carrier Frequency-Division Multiple Access ou SC-FDMA).

O espectro de frequência disponível para o LTE é dividido em diversos canais, que podem ter papel de transmitir mensagens de controle para gerenciar os recursos da rede, ou simplesmente enviar dados. Dentre os diversos canais do LTE, há o canal físico de acesso aleatório (Physical Random Access Channel ou PRACH), no qual os UEs podem iniciar o procedimento de processo aleatório em um meio compartilhado.

Com a release 10 [Ghosh et al. 2010], novos mecanismos permitiram o LTE ter um aumento da vazão e melhor eficiência espectral quando comparado com o release 8. Dentre os mecanismos que permitiram essa melhoria estão a agregação de portadoras (Carrier aggregation), que facilita o uso de uma maior largura de banda para transferência de dados, o método de múltiplas entradas e múltiplas saídas (Multiple-Input MultipleOutput ou MIMO) de ordem superior, que permite a transmissão e recepção de mais de um fluxo de dados.

\subsection{Procedimento de acesso aleatório}

O procedimento de acesso aleatório é realizado pelo UE quando: o UE está fazendo uma conexão inicial com a rede; no restabelecimento de conexão mediante falha de rádio; quando o UE não está sincronizado com o eNB; quando troca-se o eNB provendo serviço (handover).

Há dois tipos de procedimento de acesso aleatório[3GPP 2017]: livre de contenção e baseado em contenção. O primeiro é usado para realizar o handover ou quando há dados para serem recebidos no downlink mas houve perda de sincronização, enquanto o segundo é usado para os outros casos. O procedimento de acesso aleatório baseado em contenção pode ser visto na Figura 1 a seguir.

Figure 1. Procedimento de Acesso Aleatório baseado em colisão

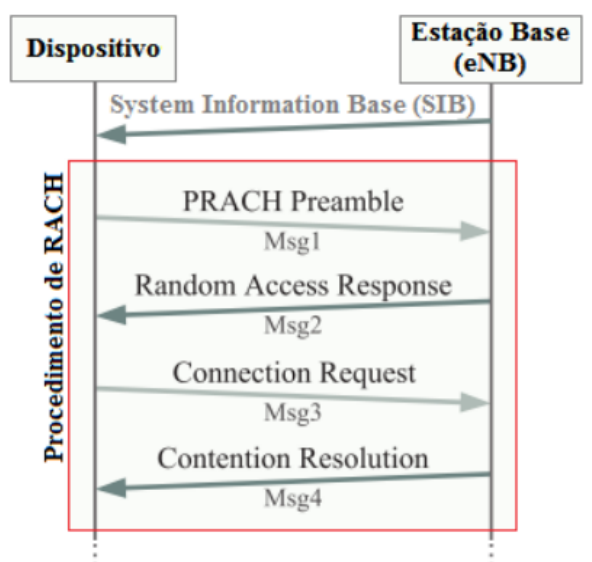




\subsubsection{Passos do procedimento de acesso aleatório baseado em colisão}

O procedimento de acesso aleatório baseado em colisão é aquele onde dois ou mais UEs podem transmitir uma mesma mensagem ao mesmo tempo. Este procedimento pode ser descrito em 4 passos:

1. UE envia uma assinatura (preâmbulo) para o eNB, indicando a intenção de se conectar na rede. Caso dois UEs enviem o mesmo preâmbulo, ocorrerá uma colisão.

2. O eNB responde com um identificador temporário e aloca recursos para que o UE transmita uma mensagem.

3. O UE envia um pedido de conexão, o (RRC connection request). Indicando o motivo pelo qual o UE deseja se conectar.

4. eNB envia uma mensagem com um novo identificador. Caso haja colisão, os UEs descobrem que suas mensagens colidiram neste momento e devem reiniciar o procedimento após um certo intervalo de tempo.

\subsection{Machine to Machine}

A comunicação Machine to Machine (M2M) permite que dispositivos interajam com pouca ou nenhuma intervenção humana. O M2M implementa aplicações em diversas área, como transportes, saúde, energia, e cidades inteligentes. Os dispositivos podem estar em diversos ambientes - carros, torres de celulares, farol de trânsito - e em grandes quantidades, todos conectados à Internet, formando uma rede de dispositivos conhecida como Internet das Coisas (Internet of Things ou IoT).

Os dispositivos M2M podem ser divididos em duas categorias, os de monitoramento de dados e os de troca de dados. Dispositivos de monitoramento apenas enviam dados. Nessa categoria, os dispositivos são usados como sensores para reportar dados a serem processados. Na outra categoria, os dispositivos também recebem uma resposta vinda do servidor. Dados são enviados ao servidor, onde eles são processados e enviados novamente para o UE, com instruções de como o dispositivo deve se comportar após o processamento dos dados.

$\mathrm{O}$ uso da interface sem fio LTE tem diversas vantagens para aplicações M2M. O LTE permite a implementação de dispositivos na maioria das áreas urbanas e rurais. A infraestrutura bem fundamentada do LTE torna desnecessário a implementação de estações bases exclusivas para comunicação M2M. Provedores de serviços podem aproveitar melhor seus recursos ao compartilhar as frequências de banda pouco utilizados por usuários comuns com os dispositivos M2M.

No entanto, as redes LTE são otimizadas para comunicação do tipo Human to Human $(\mathrm{H} 2 \mathrm{H})$ e há diversos problemas para dispositivos M2M ao acessar redes móveis. Diferente do tráfego $\mathrm{H} 2 \mathrm{H}$, que é caracterizado pela transmissão periódica com alta taxas de dados, M2M normalmente possui transmissão mais frequentes com baixa taxa de dados. Há também o problema com dispositivos que usam bateria, pois utilizam grande parte da sua carga com a transmissão de dados.

\section{Ferramenta simulador LTE-Sim}

O LTE-Sim[Piro et al. 2011] é um framework de código aberto para simulação de redes de acesso LTE, desenvolvido na Universidade Politécnica de Bari. A versão disponibilizada 
pelos criadores inclui diversas funcionalidades de redes LTE, além do UE e eNB, ela também inclui componentes do EPC. Ele suporta gerenciamento de QoS, ambientes com múltiplos usuários, mobilidade de usuários, procedimento de handover e técnicas de reuso de frequência. Quatro tipo de módulos da rede são modelados: UE, eNB, Home eNB (HeNB) e Mobility Management Entity/Gateway (MME/GW). Quatro tipo de geradores de tráfego na camada de aplicação estão implementados e o gerenciamento de bearer é suportado. Por fim, as estratégias de escalonamento mais conhecidas e modelos de propagação para camada física foram desenvolvidos.

O LTE-Sim é um simulador escrito em linguagem $\mathrm{C}++$ e orientado a cenários. Cenários são arquivos que fornecem os parâmetros de simulação, como o número de usuários, largura de banda de upload e download, métodos de acesso aleatório, etc. $\mathrm{O}$ simulador executa o cenário e informa sobre o que está ocorrendo com cada nó simulado (eNB e UE) em cada subframe, envio e recepção de mensagens também são informados. Scripts em Python foram usados para extrair informações da saída do simulador, gerar gráficos e analisar os resultados.

\section{Extensão do simulador}

\subsection{Procedimento de Acesso Aleatório}

O procedimento de acesso aleatório definido nas especificações do 3GPP foi descrito em 2.1, mas em [Zhang et al. 2015] é proposto um novo procedimento baseado em colisão para comunicação M2M, que foi implementado no simulador. Nele, a colisão é detectada antecipadamente, o que faz com que os UEs envolvidos na colisão finalizem o procedimento mais rapidamente e iniciem uma nova tentativa, economizando tempo e recursos. Pode-se ver na Figura 2 que o UE ao enviar o preâmbulo, envia também seu ID único. No procedimento tradicional, não haveria colisão no envio do mesmo preâmbulo por dois UEs distintos por se tratar da mesma mensagem. No procedimento proposto, o ID único causa a colisão, o que impede que o UE decodifique a mensagem e não responda. Sem resposta, os UEs esperarão um certo tempo até tentarem realizar o procedimento novamente.

\subsection{Modelos de tráfego em comunicação M2M}

Modelos de tráfego são modelos matemáticos para representar o tráfego de dados de uma determinado tipo de aplicação. Modelos possuem parâmetros como quantidade de pacotes enviados, número de bits por pacote, tempo de chegada do pacote, etc. Os modelos de tráfego mais comuns para comunicação M2M são:

\section{Event Driven}

Modelo no qual o dispositivo M2M envia uma mensagem para o eNB quando há um evento ocorrendo. Tem maior importância, em relação aos outros tipos de aplicações dependendo da magnitude do evento. Por exemplo, um contador de ritmo cardíaco que monitora uma parada cardíaca em um paciente. Enquanto o evento não ocorre, o dispositivo se mantém desconectado da rede, mantendo-se

\section{Time Driven} em estado de espera.

Semelhante ao Event Driven, no entanto, as aplicações deste tipo enviam dados periodicamente para um servidor M2M. Um exemplo deste tipo de tráfego são medidores de água ou eletricidade com medições são coletadas mensalmente. 
Figure 2. Esquema proposto de procedimento de acesso aleatório para comunicação M2M.

M2M UE

\section{eNB}

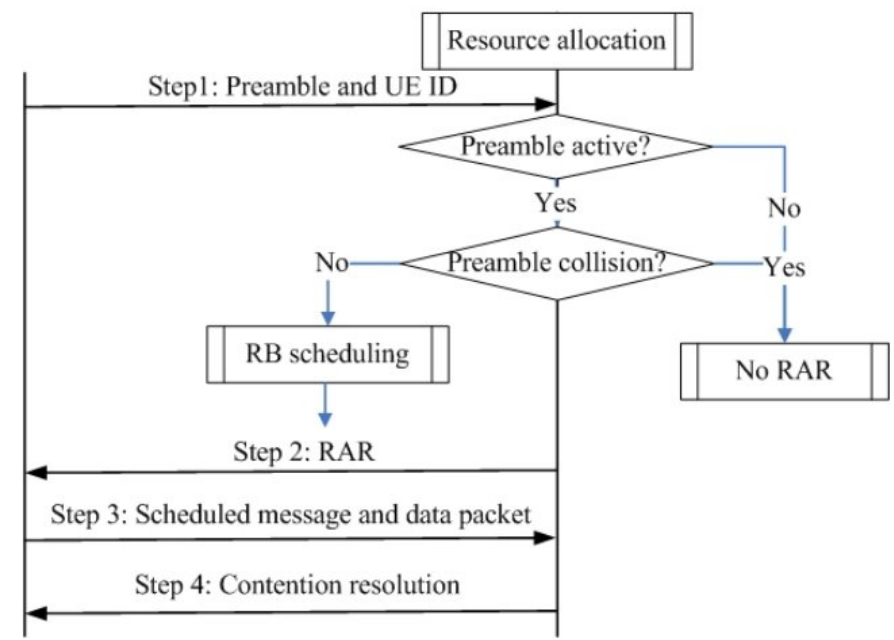

Estes modelos foram implementados no módulo para fins de estudar a comunicação M2M.

\section{Validação do módulo}

\subsection{Procedimento de Acesso Aleatório}

As classes principais modificadas para implementar o novo esquema de acesso aleatório foram a do UE e do eNB. No arquivo ue-mac-entity.cc, referente ao UE, foi incluído o novo esquema e um tempo de espera caso haja colisão de preâmbulos. No arquivo enb-mac-entity.cc, referente ao eNB, caso o eNB detecte uma colisão na transmissão do preâmbulo, ele não envia a resposta aos UEs. Os UEs tem um tempo aleatório de espera, para que não façam uma nova tentativa simultâneo.

Figure 3. Número de colisões durante a mensagem 3 do procedimento de acesso aleatório.

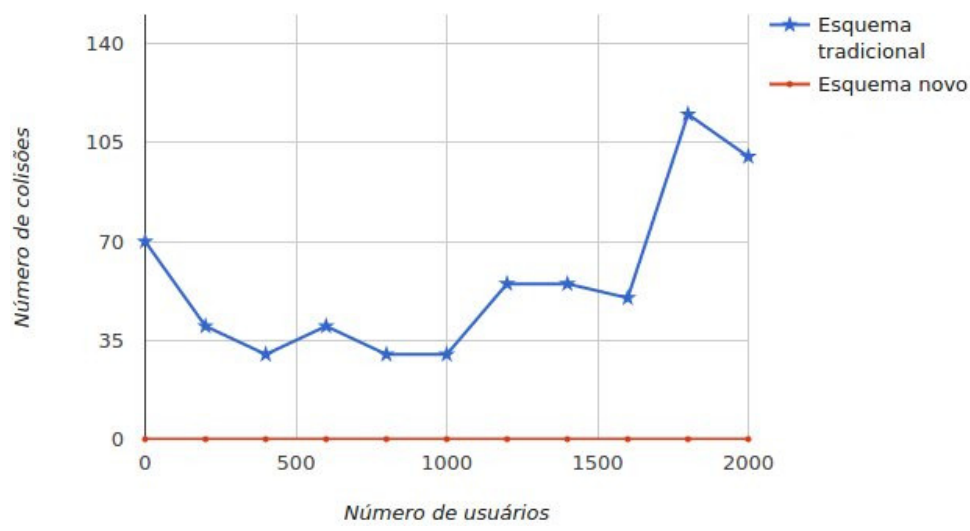

Na Figura 3, há uma comparação entre o esquema convencional [3GPP 2013] e o proposto em [Zhang et al. 2015]. No esquema novo, como a colisão é detectada antecipadamente, dois UEs diferentes não recebem o mesmo identificador do eNB, logo não há colisão. 


\subsection{Modelos de tráfego em comunicação M2M}

A implementação do modelo Time Driven foi feita a partir da criação de dois arquivos no simulador, além de modificações menores em outros arquivos para incluir o novo modelo. Foi criada uma classe chamada TimeDriven.cpp que possui as funções necessárias para o funcionamento do modelo, funções para iniciar a aplicação, escalonar o modelo no simulador e enviar pacotes. O outro arquivo importante da implementação é o cenarioTeste.h, onde está definido a simulação, o eNB, os UEs, o tamanho de banda de uplink e downlink e o intervalo de envio entre os pacotes.

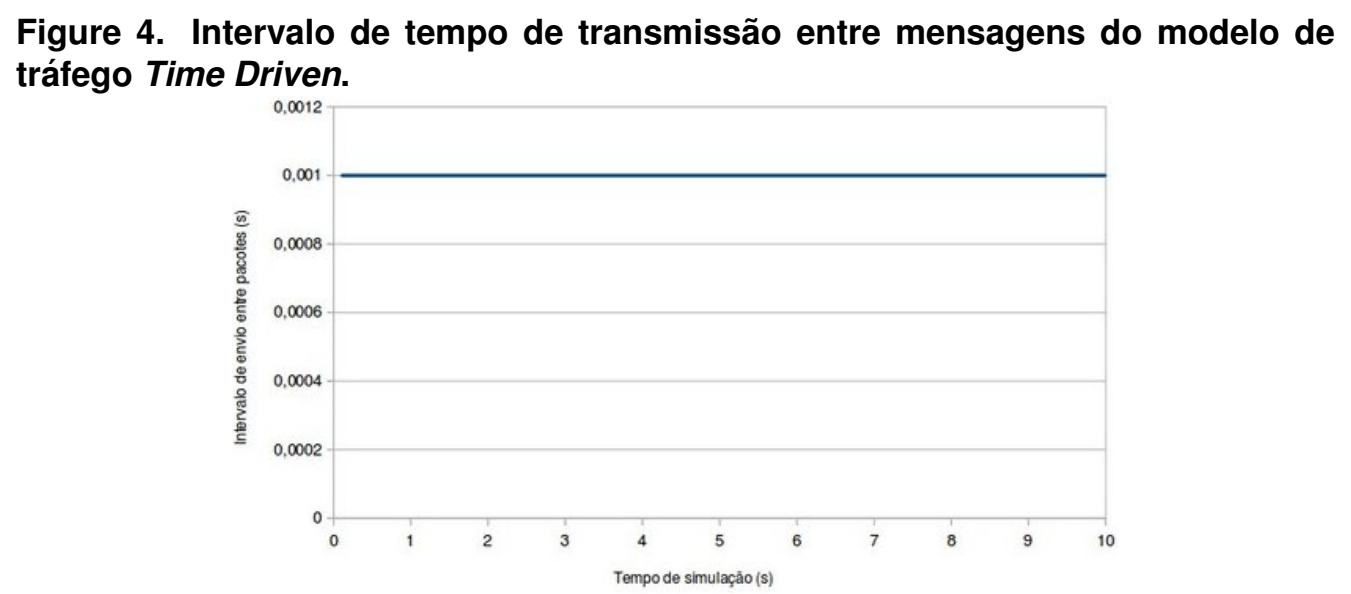

A Figura 4 representa o cenarioTeste. $h$ sendo executado. A simulação foi definida para iniciar a 0.1 segundo e terminar em 10 segundos, neste intervalo o UE enviou 1 pacote ao eNB a cada 1 milissegundo. O gráfico representa o intervalo de envio entre um pacote em um certo tempo em relação ao pacote seguinte. $O$ valor constante neste intervalo confirma que o modelo está de acordo com o esperado, pois o intervalo de tempo entre as transmissões de pacotes é sempre o mesmo. Isso pode ser verificado ao se analisar o número de pacotes enviados. A simulação durou 9900 milissegundos e foram enviados 9900 pacotes para o eNB, ou seja, 1 pacote a cada milissegundo.

O modelo Event Driven foi implementado de maneira semelhante, uma classe foi criada para incluir seus métodos, o EventDriven.cpp e outra para testar o modelo, o cenarioEventDriven.h. A diferença da implementação está no tempo de envio de pacotes, no Event Driven o evento pode ocorrer aleatoriamente, portanto a distribuição de Poisson [Ross 2014] foi utilizada para simular a ocorrência de eventos. Usando uma variável aleatória e a média de ocorrência de eventos por um certo período de tempo, pode-se utilizar a fórmula a seguir como um modelo para simular o intervalo de espera de envio entre pacotes do UE:

$$
t=\frac{\ln u}{\lambda}
$$

Onde $\lambda$ é média de eventos e $u$ é um valor aleatório da distribuição uniforme de $[0,1]$. t retorna um tempo aleatório de para a ocorrência do próximo evento, tempo este próximo da média dada.

A Figura 5 mostra a execução do cenário de testes do Event Driven. Na simulação, foi definido um evento que ocorre em média 2 vezes por segundo em uma simulação de 
Figure 5. Intervalo de tempo entre mensagens do modelo de tráfego Event Driven.

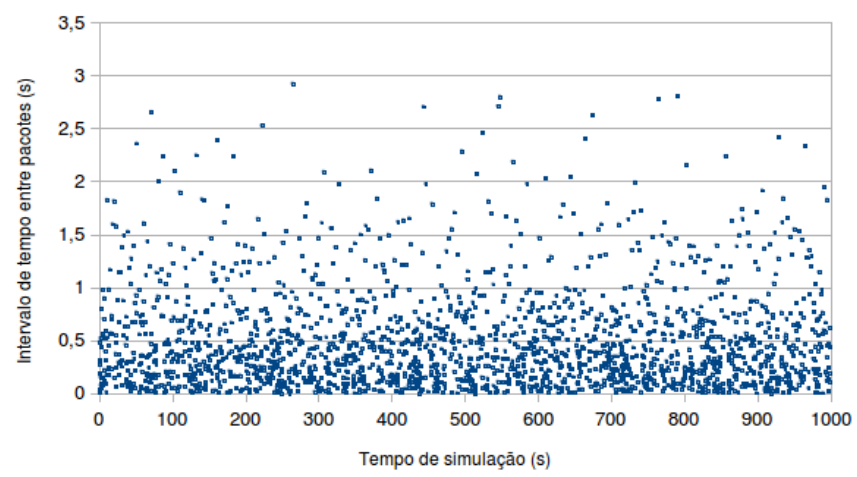

1000 segundos. No gráfico, os valores representam o intervalo de envio entre um pacote e o pacote seguinte e os valores estão próximos do esperado, 2 eventos por segundo. A quantidade de pacotes enviados também está próxima do esperado, em 1000 segundos foram enviados 2091 pacotes pelo UE ao eNB, dando uma média de 2,091 eventos por segundo.

\section{Publicações}

O trabalho realizado na Iniciação Científica foi parte de duas publicações [d. Andrade et al. 2017, Andrade et al. 2018], ambas classificadas como QUALIS A1. O valor do fator de impacto da IEEE Communications Magazine é 10.4. O módulo desenvolvido permitiu também que se fizessem diversos estudos e artigos [Astudillo et al. 2017, de Andrade et al. 2016].

\section{Conclusão}

O objetivo do projeto é de ampliar as funcionalidades do simulador ao realizar um estudo de redes LTE. O módulo criado conta com funcionalidades básicas para se simular aplicações M2M no LTE-Sim. A comparação de esquemas de acesso aleatório buscou encontrar soluções para o problema que o M2M encontra ao usar o LTE, com a redução de colisões e economia de recursos, o novo esquema pode ser desenvolvido para que se torne um substituto do esquema tradicional. O módulo implementa diversas funcionalidades novas para o simulador, permitindo que novos estudos sobre redes LTE sejam realizado.

\section{References}

3GPP (2013). Evolved Universal Terrestrial Radio Access (E-UTRA); Medium Access Control (MAC) protocol specification. TS 36.321, 3rd Generation Partnership Project (3GPP).

3GPP (2017). Evolved Universal Terrestrial Radio Access (E-UTRA); Medium Access Control (MAC) protocol specification. Technical Specification (TS) 36.321, 3rd Generation Partnership Project (3GPP). Version 14.2.1.

Andrade, T. C. P., Sekijima, L. R., and da Fonseca, N. (2018). A cluster-based randomaccess scheme for lte/lte-a networks supporting massive machine-type communications. In 2018 IEEE International Conference on Communications (ICC), volume 1, pages $1-6$. 
Astudillo, C. A., de Andrade, T. P. C., and da Fonseca, N. L. S. (2017). Allocation of control resources with preamble priority awareness for human and machine type communications in lte-advanced networks. In 2017 IEEE International Conference on Communications (ICC), pages 1-6.

d. Andrade, T. P. C., Astudillo, C. A., Sekijima, L. R., and d. Fonseca, N. L. S. (2017). The random access procedure in long term evolution networks for the internet of things. IEEE Communications Magazine, 55(3):124-131.

de Andrade, T. P. C., Astudillo, C. A., and da Fonseca, N. L. S. (2016). Allocation of control resources for machine-to-machine and human-to-human communications over lte/lte-a networks. IEEE Internet of Things Journal, 3(3):366-377.

Ericsson (2017). Mobility report. Technical report.

Ghosh, A., Ratasuk, R., Mondal, B., Mangalvedhe, N., and Thomas, T. (2010). Lteadvanced: next-generation wireless broadband technology. IEEE wireless communications, 17(3):10-22.

Ikuno, J. C., Wrulich, M., and Rupp, M. (2010). System level simulation of lte networks. In 2010 IEEE 71st Vehicular Technology Conference, pages 1-5.

Martín-Sacristán, D., Monserrat, J. F., Cabrejas-Peñuelas, J., Calabuig, D., Garrigas, S., and Cardona, N. (2009). On the way towards fourth-generation mobile: 3gpp lte and lte-advanced. EURASIP Journal on Wireless Communications and Networking, 2009(1):354089.

Mehlführer, C., Wrulich, M., Ikuno, J. C., Bosanska, D., and Rupp, M. (2009). Simulating the long term evolution physical layer. In 2009 17th European Signal Processing Conference, pages 1471-1478.

Piro, G., Grieco, L. A., Boggia, G., Capozzi, F., and Camarda, P. (2011). Simulating lte cellular systems: An open-source framework. IEEE Transactions on Vehicular Technology, 60(2):498-513.

Ross, S. (2014). A first course in probability. Pearson Education, Inc, Upper Saddle River, New Jersey.

Rupp, M., Schwarz, S., and Taranetz, M. (2016). The Vienna LTE-Advanced Simulators: Up and Downlink, Link and System Level Simulation. Signals and Communication Technology. Springer Singapore, 1 edition.

Sesia, S., Toufik, I., and Baker, M. (2011). LTE - The UMTS Long Term Evolution: From Theory to Practice. Wiley.

Zhang, N., Kang, G., Wang, J., Guo, Y., and Labeau, F. (2015). Resource allocation in a new random access for $\mathrm{m} 2 \mathrm{~m}$ communications. IEEE Communications Letters, 19(5):843-846. 